\title{
Cylindrical and Powell liquid crystal lenses with positive-negative optical power
}

\author{
J. F. Algorri, P. Morawiak, D. C. Zografopoulos, N. Bennis, A. Spadlo, L. Rodríguez-Cobo, L. R. Jaroszewicz, \\ J. M. Sánchez-Pena, and J. M. López-Higuera
}

\begin{abstract}
We demonstrate a technique to engineer cylindrical and Powell liquid crystal lenses with positive or negative optical power. The device is based on two indium-tin-oxide electrode combs and a microstructured voltage transmission electrode. The technique features the advantages of a multielectrode lens, albeit using a single lithographic step and only two voltage sources. Extensive control of the phase profile across the device active area is demonstrated, achieving both positive and negative optical power. The lens aperture is not constrained by the geometrical parameters and can be scaled to larger values.
\end{abstract}

Index Terms-Cylindrical lenses, Powell lenses, liquid crystals.

\section{INTRODUCTION}

Apart from classic display applications, liquid crystals (LC) are widely employed in optics and photonics, e.g., as active materials in polarization control and filtering [1]. LC-tunable components provide several advantages, such as high tunability, low cost, and compact design. Among the various LCbased applications, phase modulation is of the most important [2]. Despite the availability of commercial LC phase modulators, these tend to be bulky and both their price and complexity are usually high. In this regard, different topologies have been recently proposed to simplify the issue and obtain smarter optical components. Examples include lenses for ophthalmic applications [3] and zooming [4], microlenses for autostereoscopic displays [5] and integral imaging [6], optical vortices [7]-[9], and beam steerers [10].

One key issue of LC-based devices is how to increase their active area without compromising their performance

Manuscript submitted June 24, 2020. (Corresponding author: J. F. Algorri) J. F. Algorri is with the Photonics Engineering Group, University of Cantabria, 39005, Santander, Spain. (e-mail: algorrijf@unican.es)

P. Morawiak, N. Bennis, A. Spadlo, and L. R. Jaroszqwicz are with the Faculty of Advanced Technologies and Chemistry, Military University of Technology, 2 gen. S. Kaliskiego St., Warsaw 00-908, Poland.

D. C. Zografopoulos is with the Consiglio Nazionale delle Ricerche, Istituto per la Microelettronica e Microsistemi, 00133 Rome, Italy.

L. Rodríguez-Cobo is with CIBER-bbn, Instituto de Salud Carlos III, 28029, Madrid, Spain.

J. M. Sánchez-Pena is with the Department of Electronic Technology, Carlos III University, Madrid 28911, Spain.

J. M. López-Higuera is with the Photonics Engineering Group, University of Cantabria, 39005, Santander, Spain, the CIBER-bbn, Instituto de Salud, 28029, Madrid, Spain, and the Instituto de Investigación Sanitaria Valdecilla (IDIVAL), 39011, Santander, Spain.

This work was supported by the Comunidad de Madrid and FEDER Program (S2018/NMT-4326), the Ministerio de Economía y Competitividad of Spain (TEC2013-47342-C2-2-R and TEC2016-76021-C2-2-R), the FEDER/Ministerio de Ciencia, Innovación y Universidades and Agencia Estatal de Investigación (RTC2017-6321-1 and PID2019-107270RB-C21), the Ministry of National Defense of Poland (GBMON/13-995/2018/WAT), and the Military University of Technology (Grant no. 23-895). and overall complexity. Common solutions are based on multielectrode designs, hole-patterned electrodes and modal control. The first one relies on a large number of electrodes for local voltage application. Its main associated drawback is the complex fabrication process and the requirement of numerous voltage sources [11]. The hole-patterned technique is a simple means of obtaining LC lenses. Nevertheless, it is limited to small apertures, directly proportional to the device thickness, typically around a diameter two times the thickness [12]. Finally, modal control uses a hole-patterned electrode on which a high resistivity layer is deposited [13], thus avoiding limitations on the hole-patterned aperture size. However, this layer has to be very thin, which limits the range of suitable materials and complicates the fabrication process. Finally, extra options are based on non-uniform alignment layers, e.g., by photoalignment or photopatterning [14], [15], or the use of polymer networks [16]. Still, the associated non-uniformity can result in image degradation, whereas polymer networks often introduce excessive light scattering.

In short, it is far from trivial to obtain a design valid for any target aperture, which involves simple fabrication and voltage control scheme. Here, a novel device to generate LC lenses is proposed and experimentally demonstrated. The device is based on the operational principle of the transmission electrode technique [17], but with the particularity of rectangular apertures. It features the advantages of a multielectrode lens, but it requires only a single lithographic step of a standard indium-tin-oxide (ITO) substrate for the fabrication of the electrode configuration and only one or two driving voltage sources. The voltage and the resulting optical phase profiles are controlled by adjusting the shape of two high-resistance ITO voltage transmission electrodes. Then, a series of parallel ITO stubs distribute the voltage profile over the active area of the device. Using such simple configuration, we demonstrate the case examples of positive and negative cylindrical and Powell lenses. Nevertheless, the technique is generic and it can also be implemented for components based on other continuous spatial phase profiles.

\section{DESIGN AND OPERATING PRINCIPLE}

The proposed structure is shown in Fig. 1(a) and it is based on a nematic LC planar cell between two ITO-on-glass substrates. Alignment layers provide homogeneous LC orientation at the LC/substrate interfaces. The electrode structure is patterned on one substrate by a single lithographic step. As depicted in Fig. 1(b), this pattern consists of two main 


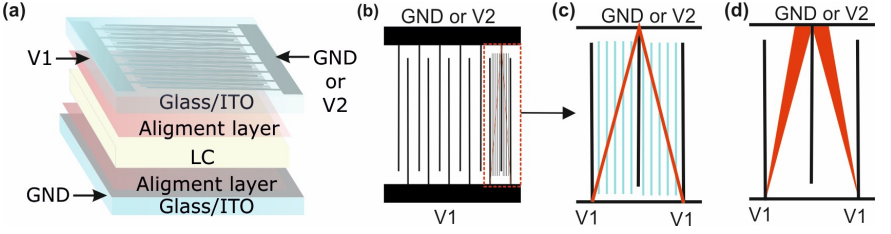

Fig. 1. (a) Schematic diagram of the proposed LC-tunable lens: a planar LC cell is formed between two standard ITO substrates, one of which is patterned with the desired electrode configuration. (b) Periodic arrangement of the ITO electrodes. (c) Zoomed period of the electrode structure. Electrodes of constant width (red) generate the target linear voltage drop profile between the two side control electrodes. The voltage profile is distributed over the entire device active area by ITO electrode stubs (cyan). (d) Configuration with linearly varying electrode width leading to a parabolic-like voltage profile.

elements, two comb electrodes, which are connected by a Vshaped voltage transmission electrode, and several parallel ITO stubs that distribute the voltage homogeneously over the active area of the device. The stubs are better discerned in Fig. 1(c), which shows a zoomed part of the periodic configuration. The parallel stubs make electrical contact with the transmission electrode only and not with the two comb electrodes, which are connected to two voltage sources $V_{1}$ and $V_{2}$.

In the device here experimentally investigated, the pitch of the electrode array, or equivalently the lens aperture, is $420 \mu \mathrm{m}$ and the total area is $33.6 \times 27 \mathrm{~mm}^{2}$. These dimensions have been selected so as to demonstrate the viability of the approach, since they cannot be realized either with modal control or hole-patterned techniques. In the modal case, the required resistivity of the control layer would be too high, with all the associated fabrication difficulties. In the case of a hole-patterned lens, a LC cell thickness around $210 \mu \mathrm{m}$ would be required, contrary to the $87 \mu \mathrm{m}$ of the demonstrated device. Thicknesses higher than $100 \mu \mathrm{m}$ are not recommended due to possible defects on the molecular alignment and high switching times. As the voltage can be fully controlled over the active area, the proposed technique can be, in principle, scaled to any aperture, since it is independent from parameters such as the LC cell thickness. It has to be noted that the optical properties, such as optical power, would be still dependent on the lens aperture.

The operating principle is based on the contribution of three physical effects. The first one is the low resistivity of the ITO electrodes, in comparison with the LC impedance. As a consequence, a continuous voltage drop is generated between the two side control electrodes. The ITO electrodes have a nominal square resistance $R_{\mathrm{sq}}=100 \Omega / \mathrm{sq}$ and a width of $10 \mu \mathrm{m}$, with a total resistance in the range of a few hundred $\mathrm{k} \Omega$, which keeps the current consumption very low. For voltage transmission electrodes of constant width, as in Fig. 1(c), the voltage profile is linear. Parabolic-like profiles are possible by employing electrodes with linearly varying width, as shown in Fig. 1(d). Figure 2 shows some voltage drop profiles for constant and linearly varying transmission electrode widths, calculated by means of the finite-element method implemented in COMSOL Multiphysics ${ }^{\mathrm{TM}}$. In the constant case the electrode width is fixed at $10 \mu \mathrm{m}$, while in the linearly varying case it increases from $10 \mu \mathrm{m}$ at the two
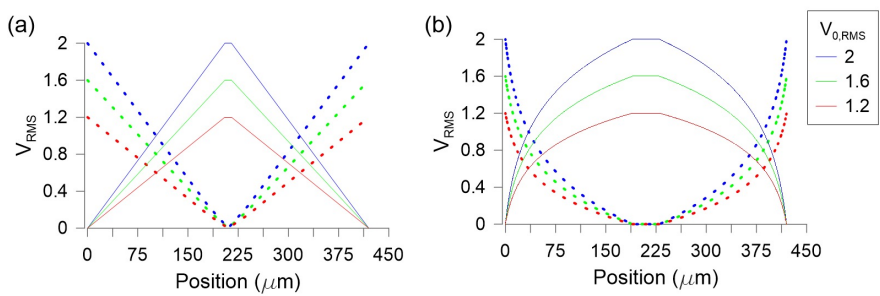

Fig. 2. Positive and negative voltage drop profiles for transmission electrodes with (a) constant and (b) linearly varying width. Continuous and dashed lines refer to a set of driving voltages $V_{2}=V_{0, \mathrm{RMS}}, V_{1}=0$ and $V_{1}=V_{0, \mathrm{RMS}}$, $V_{2}=0$, respectively, as defined in Fig. 1 .

lateral sides, i.e. at the $V_{1}$ points in Fig. 1(d), to $20 \mu \mathrm{m}$ at the opposite side.

The second effect is the electro-optic tunability of the nematic LC material. The applied voltage profile generates a Fréedericksz-like transition, which tilts the LC optical axis in the plane defined by the alignment direction and the axis perpendicular to the device. Consequently, the electrically controlled LC birefringence leads to spatial modulation of the phase accumulated of the propagating light beam, which enables the manifestation of the various target lensing effects. Finally, the third effect is the low birefringence change and hence continuity of the modulated phase profile in the gaps between adjacent parallel electrodes [17]. This is due to a reduced voltage gradient and a high aspect ratio between the LC cell thickness of $87 \mu \mathrm{m}$ and the interelectrode gap, which is equal to the electrode width, namely $10 \mu \mathrm{m}$. As a consequence, the spatial phase profile is practically continuous across the active area for the investigated values of operating voltages. This effect can be further suppressed by pushing the photolithographic resolution limit to lower values.

\section{FABRICATION}

For the fabrication, two commercial ITO-on-glass substrates were used (Glaston) with a thickness of $0.7 \mathrm{~mm}$. The ITO layer thickness was $50 \mathrm{~nm}$ and the ITO-on-substrate transparency was between 80 and $90 \%$ in the visible spectrum. The width of the transmission electrodes, stubs, and the interelectrode gap was $10 \mu \mathrm{m}$, as a safe limit for the resolution of the employed lithographic process [17]. After the exposure, the positive photoresist was developed using Microposit Developer (Shipley Far East Ltd. Japan), protecting the area of the electrode microstructure. The remaining developer was rinsed with distilled water. The substrates were dried using a pressurized $\mathrm{N}_{2}$ gun and placed on a hot stage for $30 \mathrm{~min}$ at $110^{\circ} \mathrm{C}$ to cure the photoresist. The subsequent wet etching took place in an acid bath of $\mathrm{HCl}: \mathrm{H}_{2} \mathrm{O}: \mathrm{HNO}_{3}$ at $58^{\circ} \mathrm{C}$ leading to the removal of the ITO from the exposed areas. The optimal etching time was obtained after several tests due to the complexity of the designed electrode microstructure, as it depends on both the ITO thickness and the correct proportions of the etching solution. Once the exact time is determined, no missing electrodes are observed and the samples show excellent homogeneity. 


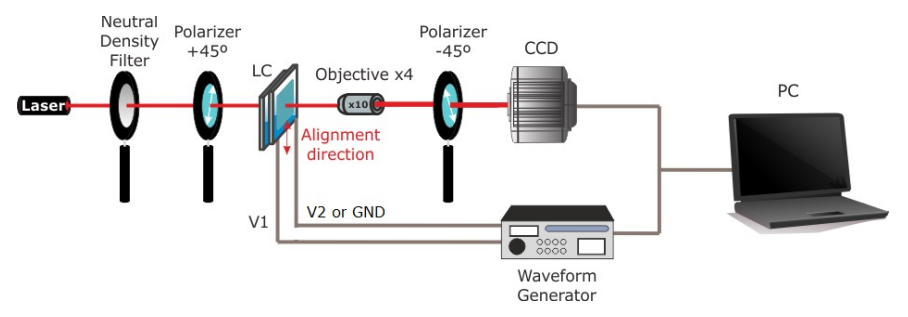

Fig. 3. Schematic diagram of the experimental setup for the measurement of the spatial phase profile generated by the investigated tunable LC lenses.
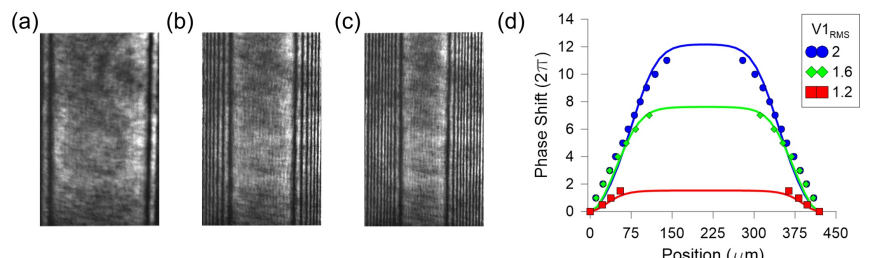

Fig. 4. (a-c) Measured interference patterns for driving voltages of $V_{2}=0$ and $V_{1}$ equal to $1.2,1.6$, and $2 \mathrm{~V}$, respectively. (d) Corresponding extracted (markers) and numerically calculated (continuous lines) phase profiles.

After the etching, the substrates were rinsed in water to prevent undercutting of the photoresist layer. The remaining photoresist was removed by immersing the samples in an appropriate solvent (Microposit Remover) for $90 \mathrm{~s}$. After the patterning process, a polyimide alignment layer was spincoated and rubbed on the patterned substrates in order to induce the preferred LC molecular orientation. Following the rubbing treatment, the two substrates were attached by using a photocurable adhesive material deposited at the edges of the cell. The LC cell was assembled by dispensing a photopolymerizable epoxy (NOA 68) seal on the perimeter of one substrate, while high precision $87 \mu \mathrm{m}$ silica sphere spacers were dispersed on the opposing substrate. The two substrates were placed with antiparallel alignment, namely with opposite rubbing directions.

Last, the cavity was infiltrated via the capillary effect with the nematic LC 6CHBT and the device was sealed by epoxy curing. The selected nematic mixture is characterized by the following parameters: density $\rho=1.01 \mathrm{~g} / \mathrm{cm}^{3}$ (at $\mathrm{T}=20^{\circ} \mathrm{C}$ ) [18], optical extraordinary and ordinary refractive indices: $n_{\mathrm{e}}=1.68$ and $n_{\mathrm{o}}=1.52(\Delta n=0.16)$ [18], low-frequency dielectric permittivities: $\varepsilon_{\perp}=5$ and $\varepsilon_{\|}=12(\Delta \varepsilon=7$, at $1 \mathrm{kHz}$ ) [18], viscosity: $\gamma=21 \mathrm{mPa} \cdot \mathrm{s}$ at $20^{\circ} \mathrm{C}$ [18], and elastic constants: $K_{11}=6.71 \mathrm{pN}, K_{22}=2.93 \mathrm{pN}$, and $K_{33}=7.38 \mathrm{pN}$ [19].

\section{EXPERIMENTAL RESULTS AND DISCUSSION}

For the experimental results, a typical optical setup is used, which employs two crossed polarizers that generate an amplitude interference pattern that describes the profile of the spatially modulated optical phase. The setup is schematically presented in Fig. 3. The first polarizer splits the impinging laser beam $(\lambda=632.8 \mathrm{~nm})$ in two orthogonal components. The first one sees the LC ordinary refractive index, while the second one is affected by the effective refractive index, which depends on the LC molecular profile and hence the applied
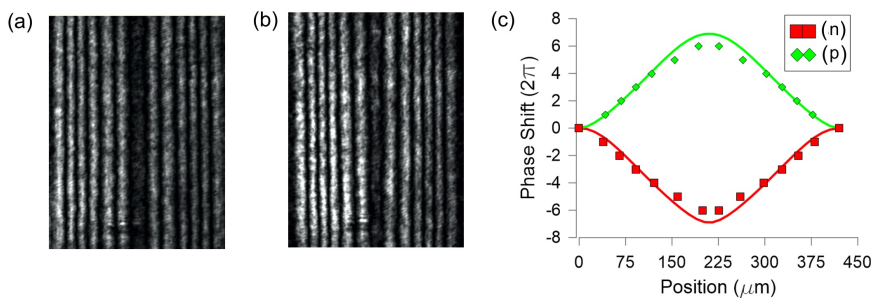

Fig. 5. Measured interference patterns for operation of the device as a Powell lens with (a) positive $\left(V_{1}=1 \mathrm{~V}, V_{2}=1.6 \mathrm{~V}\right)$ and (b) negative $\left(V_{1}=\right.$ $1.6 \mathrm{~V}, V_{2}=1 \mathrm{~V}$ ) phase profile. (c) Corresponding extracted (markers) and numerically calculated (continuous lines) phase profiles. (a)

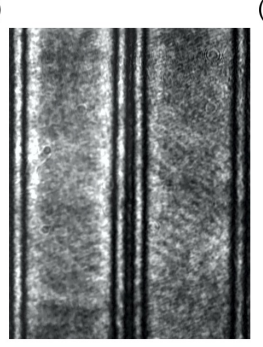

(b)

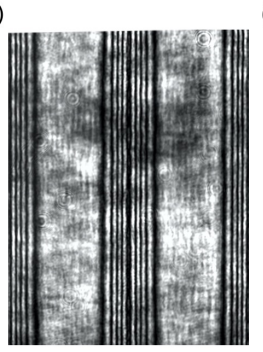

(c)

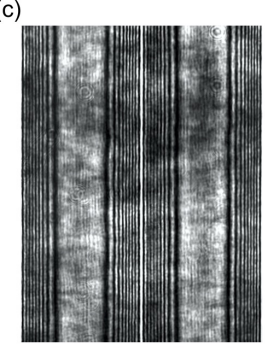

Fig. 6. Interference patterns for opposite driving signals $\left(V_{1}=-V_{2}=A\right)$ in order to obtain half-aperture lenses for $A$ equal to: (a) 1.2 , (b) 1.6 , and (c) $2 \mathrm{~V}$.

voltages. The two components arrive at the second polarizer with a relative phase shift. Shifts that are even multiples of $\pi$ are absorbed (transmittance minima), whereas phase shifts equal to odd multiples of $\pi$ pass through (transmittance maxima). As a result, an interference pattern is captured in the charged-couple device (CCD) camera.

The first experiment involves the application of only one voltage source $V_{1}\left(V_{2}=0\right)$. All the voltage signals applied in the experiments were sinusoidal at $1 \mathrm{kHz}$. This configuration creates linearly varying voltage profiles, as shown in the dashed lines of Fig. 2(a). In the central zone there is an extended area in which the applied voltage stays below the switching voltage threshold in the planar LC cell, which is approximately $1 \mathrm{~V}$. In that zone no optical phase modulation occurs. This zero-switching zone shrinks as the applied voltage increases, as demonstrated in Fig. 4(a-c). The generated phase profiles are then extracted and directly compared to LC simulations, performed over the 2D cross-section of the device, for the considered voltage profiles [20]. As these profiles cross through the LC switching threshold in their spatial variation, the resulting LC profile takes place in the most sensitive part of the voltage-dependent LC birefringence curve, i.e. close to the threshold and far below the saturation voltage. This is the reason why they were selected for the validation of the theoretical model. The effective refractive index $n_{\text {eff }}$ is calculated and the modulated phase is retrieved by integration of the phase difference caused by the effective birefringence between $n_{\mathrm{eff}}$ and $n_{e}$ over the LC thickness. The numerically calculated profiles reproduce with high accuracy the experimentally measured ones. These results demonstrate the proofof-concept of the device and its capability to modulate the optical phase profile. 
The second experiment targets the generation of Powell lenses, whose operation is based on prismatic phase profiles with a curved roofline. These are obtained in the proposed device by setting one of the two voltage sources at $1 \mathrm{~V}$ and varying the other voltage source. This way, a linear voltage distribution between the switching and threshold voltages is achieved. By inverting the two voltages, one can switch between positive and negative profiles, as shown in Fig. 5 . The flat central area is minimized to a small residual area that is required for the Powell lens.

The last experiment demonstrates a configuration that reduces by half the lens aperture. To this end, phase-shifted voltage signals are applied with equal amplitude but opposite sign, that linearly distribute the voltage over the lens aperture from $A$ to $-A$ volts. Since the LC is affected by the absolute value of the applied voltage, the optical phase profile is similar to the first experiment but having half the aperture. This is observed in the phase profiles of Fig. 6, which have double periodicity (half pitch) of those studied in Fig. 4, although they were measured for the same voltage amplitude values. The generated voltage profiles are independent of the lens aperture. Therefore, by properly scaling the electrode structure, any aperture would be in principle possible by the proposed technique, thus enabling multiple possibilities in the design of LC lenses, such as spherical, axicons, etc. As a final remark, the switching times of all the cases presented were in the order of a few seconds, which is expected for nematic LC cells of the considered thickness of $87 \mu \mathrm{m}$.

\section{CONCLusions}

In summary, we have demonstrated LC-tunable cylindrical and Powell lenses with positive-negative optical powers by employing a novel technique, which is characterized by simple fabrication and voltage control. The device aperture can be in principle scaled to arbitrary values. Overall, the proposed device outperforms previous approaches and could open new avenues of research in LC-based optical phase modulation.

\section{REFERENCES}

[1] D. C. Zografopoulos, R. Asquini, E. E. Kriezis, A. d'Alessandro, and R. Beccherelli, "Guided-wave liquid-crystal photonics," Lab Chip, vol. 12, no. 19, pp. 3598-3610, 2012.

[2] J. M. Otón, E. Otón, X. Quintana, and M. A. Geday, "Liquid-crystal phase-only devices," J. Mol. Liq., vol. 267, pp. 469-483, 2018.

[3] J. Bao-Guang, C. Zhao-Liang, M. Quan-Quan, H. Li-Fa, L. Chao, and $\mathrm{X}$. Li, "Simulated human eye retina adaptive optics imaging system based on a liquid crystal on silicon device," Chin. Phys. B, vol. 17, no. 12, pp. 4529-4532, 2008.

[4] P. Valley, M. R. Dodge, J. Schwiegerling, G. Peyman, and N. Peyghambarian, "Nonmechanical bifocal zoom telescope," Opt. Lett., vol. 35, no. 15 , pp. $2582-2584,2010$.

[5] J. Algorri, V. Urruchi, B. García-Cámara, and J. Sánchez-Pena, "Liquid crystal microlenses for autostereoscopic displays," Materials, vol. 9, no. 1, art. no. 36, 2016.

[6] J. F. Algorri, V. Urruchi, N. Bennis, P. Morawiak, J. M. Sánchez-Pena, and J. M. Otón, "Liquid crystal spherical microlens array with high fill factor and optical power," Opt. Express, vol. 25, no. 2, pp. 605-614, 2017.

[7] J. Albero, P. Garcia-Martinez, N. Bennis, E. Oton, B. Cerrolaza, I. Moreno, and J. A. Davis, "Liquid crystal devices for the reconfigurable generation of optical vortices," J. Lightwave Technol., vol. 30, no. 18, pp. 3055-3060, 2012.
[8] J. F. Algorri, V. Urruchi, B. Garcia-Camara, and J. M. Sanchez-Pena, "Generation of optical vortices by an ideal liquid crystal spiral phase plate," IEEE Electron Device Lett., vol. 35, no. 8, pp. 856-858, 2014.

[9] M. Caño-García, X. Quintana, J. M. Otón, and M. A. Geday, "Dynamic multilevel spiral phase plate generator," Sci. Rep., vol. 8, no. 1, art. no. 15804, 2018.

[10] E. Oton, P. Morawiak, R. Mazur, X. Quintana, M. A. Geday, J. M. Oton, and W. Piecek, "Diffractive and refractive liquid crystal devices based on multilayer matrices," J. Lightwave Technol., vol. 37, no. 9, pp. 2086-2093, 2019.

[11] L. Li, D. Bryant, and P. J. Bos, "Liquid crystal lens with concentric electrodes and inter-electrode resistors," Liq. Cryst. Rev., vol. 2, no. 2, pp. 130-154, 2014.

[12] J. F. Algorri, V. Urruchi, N. Bennis, and J. M. Sanchez-Pena, "Using an analytical model to design liquid crystal microlenses," IEEE Photon. Technol. Lett., vol. 26, no. 8, pp. 793-796, 2014.

[13] J. F. Algorri, G. D. Love, and V. Urruchi, "Modal liquid crystal array of optical elements," Opt. Express, vol. 21, no. 21, pp. 24 809-24 818, 2013.

[14] X.-Q. Wang, A. M.-W. Tam, S.-Z. Jia, Q. Zhang, X.-Y. Chen, Y.-F. Xiong, Q. Zhang, Z. Liu, V. G. Chigrinov, H.-S. Kwok, and D. Shen, "Low-voltage-driven smart glass based on micro-patterned liquid crystal Fresnel lenses," Appl. Opt., vol. 58, no. 4, pp. 1146-1151, 2019.

[15] Y. Ma, A. M. W. Tam, X. T. Gan, L. Y. Shi, A. K. Srivastava, V. G. Chigrinov, H. S. Kwok, and J. L. Zhao, "Fast switching ferroelectric liquid crystal Pancharatnam-Berry lens," Opt. Express, vol. 27, no. 7, pp. 10079-10086, 2019.

[16] T. A. Sergan, V. Sergan, R. Herrera, L. Lu, P. J. Bos, and E. V. Sergan, "In situ control of surface molecular order in liquid crystals using a localised polymer network and its application to electro-optical devices," Liq. Cryst., vol. 40, no. 1, pp. 72-82, 2013.

[17] J. F. Algorri, P. Morawiak, N. Bennis, D. C. Zografopoulos, V. Urruchi, L. Rodríguez-Cobo, L. R. Jaroszewicz, J. M. Sánchez-Pena, and J. M. López-Higuera, "Positive-negative tunable liquid crystal lenses based on a microstructured transmission line," Sci. Rep., vol. 10, art. no. 10153, 2020.

[18] R. Dabrowski, J. Dziaduszek, and T. Szczucinskl, "4-trans-4'-nalkylcyclohezxyl/isothiocyanatobenzenes a new class of low-melting stable nematics," Mol. Cryst. Liq. Cryst., vol. 102, no. 5, pp. 155-160, 1984.

[19] R. Buchecker and M. Schadt, "Synthesis, physical properties and structural relationships of new, end-chain substituted nematic liquid crystals," Mol. Cryst. Liq. Cryst., vol. 149, no. 1, pp. 359-373, 1987.

[20] D. C. Zografopoulos, R. Beccherelli, and E. E. Kriezis, "Beam-splitter switches based on zenithal bistable liquid-crystal gratings," Phys. Rev. $E$, vol. 90, no. 4, art. no. 042503, 2014. 